Relations industrielles

Industrial Relations

\title{
T. TOMANDL, K. FUERBOECK : Social Partnership - The Austrian System of Industrial Relations and Social Insurance. Ithaca, N.Y., ILR Press, 1986, 165 pp., ISBN 0-87546-116-6
}

\section{Pierre Verge}

Volume 42, numéro 3, 1987

URI : https://id.erudit.org/iderudit/050351ar

DOI : https://doi.org/10.7202/050351ar

Aller au sommaire du numéro

\section{Éditeur(s)}

Département des relations industrielles de l'Université Laval

\section{ISSN}

0034-379X (imprimé)

1703-8138 (numérique)

Découvrir la revue

Citer ce compte rendu

Verge, P. (1987). Compte rendu de [T. TOMANDL, K. FUERBOECK : Social Partnership - The Austrian System of Industrial Relations and Social Insurance. Ithaca, N.Y., ILR Press, 1986, 165 pp., ISBN 0-87546-116-6]. Relations industrielles / Industrial Relations, 42(3), 662-663. https://doi.org/10.7202/050351ar

Tous droits réservés (C) Département des relations industrielles de l'Université Laval, 1987
Ce document est protégé par la loi sur le droit d'auteur. L’utilisation des services d'Érudit (y compris la reproduction) est assujettie à sa politique d'utilisation que vous pouvez consulter en ligne.

https://apropos.erudit.org/fr/usagers/politique-dutilisation/ 
Social Partnership - The Austrian System of Industrial Relations and Social Insurance, by

T. Tomandl and K. Fuerboeck, Ithaca, ILR Press, New York State School of Industrial and Labor Relations, Cornell University, 1986, 165 pp., ISBN 0-87546-116-6

Le «partenariat social» paraît bien le trait dominant du régime autrichien de rapports économiques et sociaux, dont la présentation générale est l'objet de ce succinct ouvrage. Les orientations politiques, les décisions, procèdent, de façon caractéristique, de consensus établis par les principaux intervenants: le gouvernement et ses agences, une puissante centrale syndicale (OGB) et ses syndicats affiliés, les chambres du travail, qui regroupent obligatoirement ouvriers et employés, selon le cas, des différentes provinces, ainsi que les chambres de commerce issues du regroupement, également obligatoire, du patronat, d'après, cette fois, six grandes branches professionnelles. La représentation de ces différents corps dans la composition de multiples conseils consultatifs étatiques permet notamment l'atteinte d'un tel degré de concertation.

Dans les rapports professionnels, le rôle des différents corps est néanmoins assez spécifique. Bien que les chambres établies par l'Etat, aient le pouvoir de négocier pour leurs membres, il s'agit là, en pratique, d'une fonction syndicale, du moins pour ce qui est des employés et des ouvriers. Les rôles sont toutefois souvent inversés dans le cas du patronat: ces regroupements obligatoires que sont les chambres de commerce ont déplacé à cette fin les regroupements volontaires d'employeurs. Fort d'un taux général de soixante pour cent, le mouvement syndical, par l'entremise des quinze grands syndicats qui le composent, est en mesure de concourir à l'établissement, en fonction de différents secteurs professionnels, d'une véritable politique salariale à l'échelle du pays, que consacrent des accords collectifs. Soit dit en passant, la négociation collective demeure volontaire; elle est rarement l'occasion d'arrêts de travail significatifs et elle débouche sur des conventions qui établissent des normes minimales de travail pour l'ensemble des salariés à l'emploi d'employeurs représentés par les chambres signataires. En marge de la loi cette fois, il arrive assez souvent néanmoins que des accords d'entreprise conclus par des employeurs avec des comités d'entreprise viennent parfaire à ce niveau la portée salariale de la convention. Ces comités, formule courante de représentation directe des salariés en droit du travail européen, se distinguent des organisations syndicales, qui oeuvrent au niveau de la profession, bien que des liens étroits les unissent souvent en pratique à ces dernières. La formation d'un comité d'entreprise est obligatoire dans tout établissement comptant au moins cinq salariés. Plus essentiellement, l'employeur doit consulter le comité d'entreprise relativement aux différentes décisions qui touchent le salarié. Son concours est notamment nécessaire en matière disciplinaire; par contre, les décisions relatives aux changements technologiques, ou encore, à une fermeture d'usine, ne sont subordonnées qu'à une discussion avec le comité. Mais, à ce niveau local, le système autrichien de codétermination de la conduite de l'entreprise repose primordialement sur la présence obligatoire de représentants des salariés dans le conseil de surveillance de l'entreprise, dont il occupe le tiers des sièges. Il revient en particulier à ce comité d'élire le conseil d'administration de la compagnie.

L'ouvrage expose, toujours avec clarté et précision, plusieurs autres aspects des institutions autrichiennes du travail, dont la juridiction des tribunaux du travail, y compris des observations sur l'influence de la jurisprudence dans l'élaboration du droit du travail. Il comporte aussi une seconde dimension: l'essentiel de l'ensemble de la sécurité sociale autrichienne, soit en particulier, le régime étatique et obligatoire d'assurance-chômage et son complément, la législation visant à favoriser l'accès à l'emploi, de même que les régimes d'assurance-santé, de compensation pour les accidents du travail et prestations de retraite. Dans chaque cas, la description porte aussi bien sur les modes de financement que sur la détermination des bénéficiaires et l'étendue des prestations. 
Une impression générale de sécurité paraît se dégager pour le citoyen et la nation de ce réseau serré de rapports et de mesures complémentaires. De fait, ce «partenariat social», de l'avis des auteurs (p. 156), paraît plus important à la majorité des autrichiens que la vie politique au sens strict. Mais des spectres apparaissent également: chômage, vieillissement de la population, particulièrement à craindre pour ce qui est du financement de ce généreux système de sécurité sociale... On se soucie par ailleurs de l'effectivité des normes légale et conventionnelle face à un marché de travail au noir grandissant, de la nécessité d'intégrer dans le consensus des forces émergentes qui en sont absentes, comme telles du moins, soit les mouvements féministes et écologiques. Mais le "partenariat social» semble bien en mesure de trouver dans ses profondes racines nationales un gage de stabilité.

Pierre VERGE

Université Laval

L'organisation du travail, par Michel Paquin, Montréal, Agence d'Arc Inc., 1986, 199 pp., ISBN 2-89022-091-5

Cet ouvrage sur l'organisation du travail est destiné à ceux qui se préoccupent de savoir comment le travail est réparti dans les organisations. Il vise à contribuer à la prise de décision en matière d'organisation du travail sans privilégier toutefois une approche particulière. L'objectif est ambitieux pour un ouvrage de moins de 200 pages qui veut présenter en outre les théories du domaine et offrir au lecteur un éventail de modeles et d'instruments appropriés à diverses circonstances.

Je suis resté sur mon appétit par rapport à cet objectif tout en appréciant de façon certaine la facture et le contenu de ce petit livre. L'auteur présente de manière synthétique et relativement accessible les connaissances reçues en matière d'organisation du travail, de mesure et d'étude du travail, ainsi que certaines approches de réorganisation du travail fondées principalement sur l'analyse socio-technique. Le lecteur n'y trouvera pas d'éléments nouveaux.

La qualité de l'ouvrage tient plutôt à sa présentation: articulation des chapitres, cohérence malgré l'obligation de survoler de nombreux modeles et de nombreux auteurs et surtout, une documentation bien choisie et judicieusement citée. À ce propos, la technique de présentation utilisée est très appropriée à ce genre de volume: l'auteur présente à grands traits les paramètres d'un modèle ou d'un instrument de manière à le bien situer et à le comparer à d'autres, puis il cite immédiatement un ouvrage dans lequel une présentation détaillée du sujet est disponible. Cette technique de présentation permet au lecteur initié de parcourir rapidement le volume, de repérer les informations appropriées, d'obtenir immédiatement un résumé et le cas échéant, d'approfondir le sujet dans un autre ouvrage.

Par contre, cet ouvrage peut difficilement être perçu comme une aide à la décision. Il s'agit plutôt, à mon avis, d'une revue succinte et fort utile de la littérature, d'un aide-mémoire à la disposition des praticiens. La plupart des décideurs ont eu l'occasion de se familiariser avec ces connaissances durant leur formation ou leur expérience professionnelle. Ce livre remet celles-ci à leur disposition d'une manière beaucoup plus agréable et intégrée que ne le ferait une bibliographie commentée. Sa contribution sera peut-être d'éveiller le praticien à la lecture d'un modèle ou d'un instrument qu'il ne connaît pas, qu'il a oublié ou qu'il aurait négligé dans sa 